\title{
A Systemic Approach to Social Skills Training for Adults with Intellectual Disability: An Irish Perspective.
}

\author{
Maeve Daly ${ }^{1}$, William Kinsella ${ }^{2}$ \\ National University of Ireland, Maynooth ${ }^{l}$ \\ University College Dublin, Ireland ${ }^{2}$
}

\begin{abstract}
This study focuses on the design, implementation and evaluation of a Social Skills Training (SST) programme for adults with Intellectual Disability (ID) through a model of consultation that is frequently used by school psychologists. An SST manual of 230-pages and separate workbook were developed and made accessible at a service through a consultative process with staff and clients. The lead researcher/consultant and facilitator/main consultee co-delivered six pilot sessions with a group of adults who present with ID and co-associated conditions. Standardised assessment tools were made accessible for clients and piloted with the SST group. The preventive and remedial goals of consultation were examined. Emphasis was placed on giving voice to the clients themselves as well as members of servicestaff at different stages of the consultation. Following intervention, results showed moderate generalisation of clients' social skills to the homesetting, although nine out of ten families acknowledged subtle gains made. Process-evaluation measures used with clients informed the SST programme design and the pacing of sessions. There was improvement to the service at a systemic-level in relation to SST with the empowerment of consultee personnel within the service to continue the SST intervention. Service-staff reported high levels of treatment acceptability and satisfaction with the consultation-service. Staff expressed the intention to implement the programme under a training module in the adult training and employment centres at the service. Results support the hypothesis that consultation represents an effective model of servicedelivery when applied to a service for ID.
\end{abstract}

Keywords: Behavioural Consultation Model, Social Skills Training, Systems-work, Adults with Intellectual Disability, Adult-Education.

\section{Introduction}

This study aims to contribute to the field of Intellectual Disability (ID) with the design of a
Social Skills Training (SST) programme through consultation. Given findings that community integration is a powerful determinant of quality of life for people with ID, communication interventions need to be developed and implemented to enhance autonomy for individuals with ID across the life-span [11] [17]. Furthermore, difficulties with social interaction factors such as not maintaining friendships have been consistently found as a significant risk factor in the development of mental health problems for people with ID [10] [14]. According to Kelly, learning the necessary skills to communicate effectively in a variety of settings can have a profound effect on the quality of life for people with learning disabilities [12]. Social skills, which are central to adaptive living, are defined as specific strategies used by an individual to perform social tasks effectively and thus be judged as socially competent [2]. "Social skills training aims to increase ability to perform key social behaviours that are important in achieving success in social situations," [18].

Consultation has become one of the central roles of educational psychologists and is now considered an effective approach for providing psychoeducational services [6] [20]. Its indirect nature of service delivery, combined with its dual goals of remediation and prevention, make it an efficient service delivery approach [6]. The present study aims to approach the delivery of an SST programme for adults with ID through a model of behavioural consultation, which has proven an efficient service delivery approach in school settings [20]. According to Keith, catering for the views of the client in the process is a crucial aspect of effective intervention, which is another key goal in the present study [11].

\subsection{Consultation}

Wagner provides a broad view of consultation as a "voluntary, collaborative, non-supervisory approach, established to aid the functioning of a system and its inter-related systems" [6]. There are a number of different models of consultation proposed in the literature, with the three most frequently cited being mental health consultation, behavioural 
consultation and process consultation [6] [20]. While there are differences between models, Gutkin and Curtis identify core common characteristics; namely, an indirect service delivery, remedial and preventive goals and a collaborative consultantconsultee relationship [6]. Consultative work is seen as both a proactive and reactive service [20]. Psychologists/consultants working from an indirect service delivery model work primarily with 'caregivers' (consultees), who then work directly and intensively with clients rather than with the clients themselves. In the present study, the consultant implemented the pilot programme of SST through working alongside consultees for six sessions, with a view to empowering consultees to continue the intervention once direct support of the consultant is withdrawn [6]. A clearly articulated model of behavioural consultation (including adapted technology training) was thus applied to the design, implementation and evaluation of the SST programme at this service. The remedial and preventive aspects of consultation are particularly applicable to an educational setting for adults with ID because SST programmes are recommended as both a treatment and as a preventative strategy for adults with ID who present with comorbid psychopathology (Mc Gillivray \& McCabe,2009)

The problem-solving/behavioral model of consultation involves leading consultees through a structured problem-solving process with a four-stage framework [7]. This framework includes: problem identification; problem analysis; plan implementation; and plan evaluation. Gutkin and Curtis believe establishing priorities to be vital in the problem identification (PI) phase [6]. The problem analysis stage is a natural extension of the PI stage. Sources of information from rating scales, checklists and/or direct observations enable the consultant and consultee to form a clearer understanding of clients' difficulties. Plan Implementation has two objectives: selecting an appropriate intervention and implementing the intervention [20]. This stage involves contact between the consultant and the consultee, by which the consultant monitors intervention integrity and deals with any issues which have arisen [7]. The final evaluation phase involves the selection of outcome measures to evaluate effects of intervention including the assessment of generalisation and maintenance effects [7].

A study in Ireland evaluated the efficacy of behavioural consultation as a means of improving social skills for adolescents with Asperger Syndrome [15]. Findings showed that SST led to clinically significant improvements in students' social skills. Overall, researchers suggested that "technology training using behavioural consultation can be an acceptable method of service delivery for teachers" due to high levels of treatment acceptability and consultant effectiveness reported by all consultees [15]. Delivering SST programmes through a model of behavioural consultation has thus proven successful in schools and application of this model to a service for ID aims to enhance the effectiveness of this intervention. Therefore, the current study replicated and extended these findings through assessing the efficacy of delivering SST through a model of consultation with an adapted version of technology training at a service for ID in the Irish context.

\subsection{Intellectual disability}

Intellectual Disabilities (ID) are characterised by cognitive and adaptive limitations appearing prior to the age of 18. Deficits in both intellectual functioning and adaptive behaviour are central to definitions of ID [3]. Adaptive behavior skills fall into three core domains of functioning (Schalock as cited in Carr et al., 2007); the conceptual domain (communication, pre-academics and self-direction); the practical domain (community use, home-living, health and safety and self-care); and the social domain (leisure and social). Levels of intellectual impairment of Mild ID and Moderate ID are reflected in IQ-levels of 50-55 to approximately 70 and $35-40$ to 50-55 respectively (American Psychiatric Association) [1]. Diagnosis of ID is also based on combined levels of adaptive functioning deficits in three domains: social, conceptual and practical skills [1]. Participants in the present study have diagnoses of Mild or Moderate levels of ID, where some participants were non-verbal and also presented with further additional needs.

The use of evidence-based and inclusive instructional practices (at individual and classroom levels) is recommended for teaching skills to a population with ID [19]. Scaffolding involves engaging the person's interest, simplifying the task into a predefined number of steps, providing external encouragement for completion of the task until intrinsic motivation takes over, in addition to giving corrective feedback and modelling correct performance of incorrectly completed aspects of the task [19]. Scaffolding techniques are reflected in the SST programme manual of session plans developed by the researcher in the present study. Examples include task-analysis (where component parts of skills are broken down into a definitive sequence of steps), direct instruction (focused instruction, mastery learning, error correction and practice, discrimination training and cumulative review), cooperative learning and student-mediated strategies such as self-monitoring and self-evaluation [19]. The SST intervention is thereby tailored to the developmental levels of clients to aid progress through key phases of a learning hierarchy; 
acquisition, fluency, generalisation, adaptation and maintenance in acquiring new skills [19].

\subsection{Participatory approach to research}

With a theoretical progression into a third generation of inclusive practice; new perspectives that emphasise quality of life for individuals with ID have emerged [19]. Tools such as person-centred planning, modelled on principles of choice and freedom for the individual, are becoming more widely used within human and social service systems [11]. Findings in the quality of life literature indicate that scores on measures of quality of life increase as service models become more like those of normal community life. The transition to adulthood at the end of adolescence involves learning social, practical and vocational skills and promoting communication skills necessary to retain employment for young adults with ID [3]. However, research suggests that taking advantage of social opportunities requires appropriate communication and social skills and that communication interventions to enable assumption of adult roles need to be developed and implemented for individuals with ID [17]. Despite this, a dearth of standardised SST programmes specifically developed for adults with disabilities is found in the literature, despite on-going communication impairments experienced by this population throughout the life-span [12]. It follows thus that clinicians have an important role to play in the planning and design of effective interventions that affect change on the quality of life of individuals with ID [12]. Gaining input from clients (as adults and key stakeholders) is a crucial aspect of a participatory research approach in the design of this SST training programme. It helps to pinpoint their difficulties and ascertain the level of intervention required [11].

Designing an effective SST package that applies educational psychological techniques and promotes evidence-based practice (from later generations of inclusion) is one way to achieve this, but catering for the views of the client in the process is also a crucial aspect of effective intervention. Given the paucity of literature in relation to normed standardised measures of social skills for the population with ID, a standardised instrument is adapted and made accessible for individuals with ID [12] [18]. Following guidelines produced by the Mental Capacity Act (MCA) in the UK, researchers adapted (rather than modified) the Adaptive Behaviour Assessment System, Second Edition, Adult Form Self-Report to use with clients (adults with ID) in the present study [5] [8].

Use of inclusive instructional practices for teaching skills to a population with ID is endorsed by research [19]. Additionally, guidelines for bestpractice emphasise the importance of giving careful consideration to issues such as treatment acceptability, intervention effectiveness and use of empirically-supported approaches and resources in the design of an appropriate intervention [20]. This study aims to design an SST programme that applies educational psychological techniques and promotes evidence-based practice and one that accounts for the voice of the client in the process. To this end, semistructured questionnaires were also adapted for clients with ID to be included as accessible assessment tools in the SST manual. Further, process-evaluation measures are also used to capture views of clients throughout the pilot programme of SST and to inform the pacing of sessions.

\subsection{Social skills training}

Carr et al. point out that people with ID generally present with deficits in adapting and responding to intricate environmental cues in coping with stressful situations and avoiding interpersonal conflicts [3]. As outlined, Spence presents a framework to conceptualise social competence, which highlights a variety of factors determining social responding and how individuals behave in social situations [18]. There are a variety of cognitive (e.g. social perception skills to interpret social cues and body language), affective (e.g. anxiety, anger, depression), and environmental (environmental contingencies for social responding) factors that guide social behaviour and hence, social competence. The specific targeted areas in this study are highlighted in purple (see figure 1).

A meta-analysis of studies examining special education and related services specifically geared for individuals with ID found moderate effects for specialised interventions such as SST. However, authors concluded that positive outcomes were found when interventions were based on principles of effective instruction and structured implementation [19]. These findings suggest that tailoring programmes specifically to the developmental level of the individual and following principles of best practice is linked to its success. It is proposed that this study contributes to the field of ID research through the design, implementation and evaluation of an adapted SST programme for adults with ID. Furthermore, delivering SST programmes through a clearly articulated model of behavioural consultation was expected to enhance the effectiveness of this intervention. 


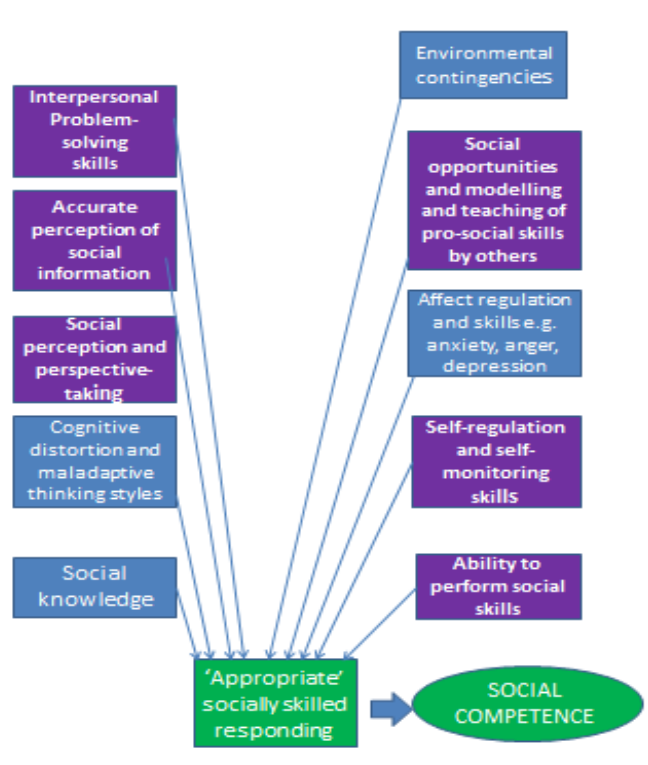

Figure 1. Some behavioural, cognitive, emotional and environmental determinants of social responding

\subsection{Research objectives}

Based on review of the literature, objectives of the current study are:

1. To adapt SST skills sessions from published SST programmes and make lessons accessible and appropriate for adults with ID and co-morbid conditions.

2. To establish whether the remedial goal of consultation was achieved:

a. To pilot six sessions out of the sixteen sessions produced for the SST programme and to examine whether the pilot programme of SST improved the social skills of adults with ID and co-morbid conditions at the service.

b. To establish whether any changes in clients' social skills generalised to their home setting.

3. To establish whether the preventive goal of consultation was achieved:

a. To take a participatory approach to research and gain clients' (adults with ID) views to inform the design of an SST programme and as part of the overall evaluation of the programme.

b. To investigate if consultees (service-staff) feel the SST programme designed through consultation meets the needs of clients and empowers staff to introduce the intervention in on-going service-delivery at a systemic level.

\section{Methodology}

\subsection{Design}

The present study employed an embedded singlecase study design because this study investigated two units of analysis in this instance of systems-work: 1) efficacy of consultation in the development of a Social Skills Training (SST) programme accessible to adults with Intellectual Disability (ID); 2) efficacy of the programme in improving their social skills while emphasising a participatory approach for the clients to the research. The exploratory nature of this study supports the use of a case study design [4].

\subsection{Setting and participants}

This study was conducted at a service for persons with ID in Dublin, Ireland. The facilitator of training programmes at one adult training centre was the main consultee, undertaking technology training directly with the lead researcher (consultant) to codeliver six pilot SST sessions. The six consultees included members of the clinical team and staff from two training centres (see figure 2). Three consultees participated in the needs analysis at pre-intervention stage. All consultees completed rating measures for treatment acceptability and consultant effectiveness at post-intervention stage. Adults with Mild and Moderate levels of ID were clients in the study and some participants were non-verbal with additional needs (Anxiety Disorder or Autism Spectrum Disorder). Clients gave their views throughout the process of programme development. Twenty-one adults consented to participate in the SST group and fourteen clients were selected for participation on the basis of priority for SST as judged by the main consultee and principal psychologist at the service.

Family members also provided their views and ratings at pre- and post-intervention stages. Data from just ten clients was included in the present study because only ten out of the fourteen families from the sample selected consented to participate in the research aspect of the SST group. A waiting list was formed for seven other clients who showed interest, but who were not initially selected for participation. 


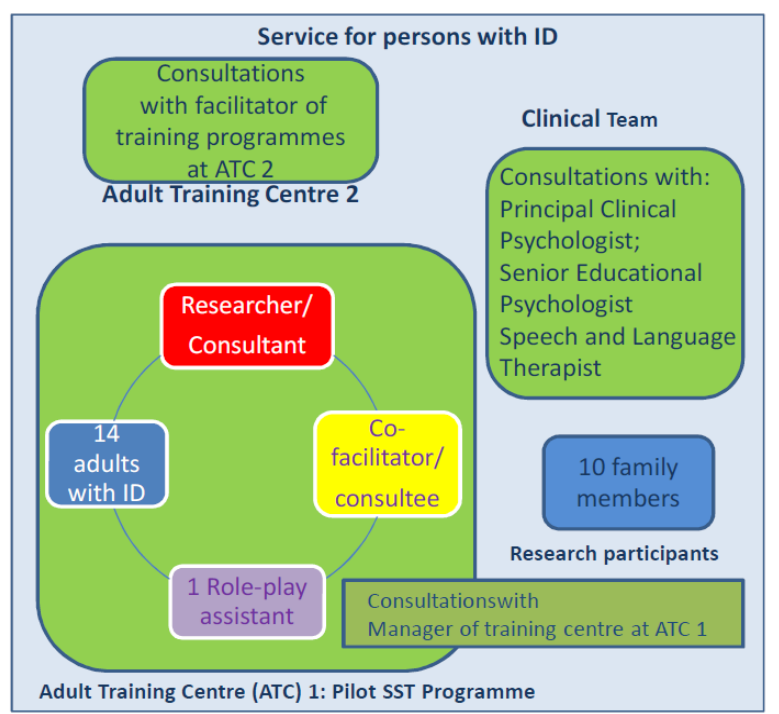

Figure 2. Setting and participants

\subsection{A Participatory Approach to Research}

In line with principles such as self-determination and choice from a quality of life perspective, all forms of communication with clients at the service, including informed consent, were made accessible for individuals with ID through the use of pictures to accompany words and sentences in line with MCA guidelines [5] [11]. This was achieved using the Boardmaker software programme (Mayer-Johnson; software for Windows), which is a Picture Communication Symbols system. Supported decision-making is emphasised for potential participants, where staff members are asked to read steps of informed consent aloud for clients, to lift any burden of reading/ decoding information when a person weighs up information to make a decision. This process is labour-intensive but it adheres closely to policies and procedures followed by the service for working with individuals with ID to enable people with learning disabilities to make valid decisions and to maximise their decision-making capacity.

2.3.1. Informed consent with clients. Open invitations for SST were issued at the adult training centre, posters were displayed and information sheets about the SST group were distributed to clients at the training centre. Clients marked a 'yes' or 'no' box at the bottom of the information sheet to indicate their interest in SST. Guidance on gaining informed consent was given to staff at the centre. Following this, staff helped potential participants to access and complete consent forms and a form for confirmation of consent was signed by the staff member for all participants.
2.3.2. Adapting the ABAS-II for clients. Test validation is the joint responsibility of the developers and its users [8]. Researchers adapted the ABAS-II (Adult Self-Report Form). MCA guidelines are adhered to, to ensure that the validity and reliability of this standardised measure is not jeopardized [5]. Boardmaker pictures and symbols were used to increase accessibility for clients with ID. To ensure items were adapted rather than modified; language is merely simplified without changing the meaning of items; pictures are used for keywords; and information is chunked. Clients sat with the researcher/support staff to complete the ABAS-II assessment with accommodations. Ideally, this work would be conducted individually with each client. Gestures and the individual's preferred method of communication were used to aid comprehension (e.g. use of sign language [lámh] signs) and such accommodations are facilitated by the support staff to facilitate the supported decision-making process [5].

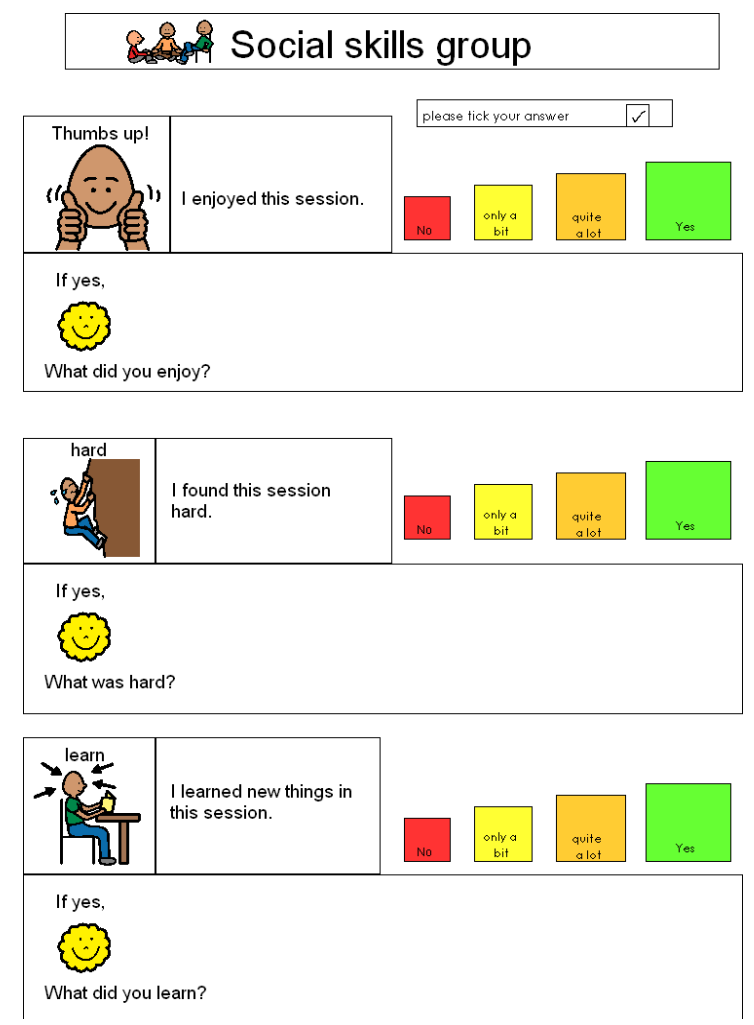

Figure 3. Process-evaluation (clients)

2.3.3. Accessible process-evaluation measures. As case studies catch the dynamics of an unfolding situation, clients with ID completed formal processevaluation procedures to provide their views on programme design [4]. At the end of each session, clients responded to three statements, 'this session was fun/this session was hard/this session was helpful' [9]. Clients were supported by facilitators to write keywords or draw pictures in response. A fourpoint colour-coded rating scale was used to remain in 
line with procedures familiar to clients at the service (see figure 3).

2.3.4. Developing accessible assessment tools. Research recommends use of assessment tools with participants at pre- and post- intervention stages of SST groups [12]. To this end, a semi-structured questionnaire from a published SST programme was adapted and made accessible for ID by the researchers. The client questionnaires are based on a combination of questions deemed useful from research and from clinical interviews provided in the Talkabout series [12] [18]. The semi-structured questionnaires were piloted at pre- and postintervention stages with three clients from the SST group and were included as accessible pre- and postassessment tools in the SST manual for use with future SST groups.

\subsection{Materials and procedure}

2.4.1. Administrative procedures. A pilot programme of six SST sessions was implemented over seven consecutive weeks from the full programme of sixteen sessions developed for future use at the service. Behavioural consultation including adapted technology training was applied to the development of the SST intervention [7]. This involved leading consultees through four phases: problem identification; problem analysis; plan implementation; and plan evaluation.

2.4.2. Problem identification. A needs analysis was conducted to establish the priority social skills needs of clients. A semi-structured interview schedule, based on adapted versions of interview schedules used in school consultation provided a framework for initial consultations with consultees [6]. Consultees and family members prioritised social skills from a menu to generate priority content for the manual (see figure 4) [12]. Skills ranged from 'how to greet others' (lower-order skills) to 'sticking up for yourself' under assertiveness skills (higher-order skills). Pre- and post- assessment questionnaires were made accessible and piloted with clients at this stage to be included in the SST manual [12].

2.4.3. Problem analysis to develop resources and measures. The researcher/consultant analysed all data collected during needs analysis to identify pertinent content for the SST manual (see table 1). A manual of sixteen lesson plans and an accompanying client workbook were compiled and made accessible for ID by the lead researcher using Boardmaker software (see figure 5). The SST programme was based on a synthesis of resources and materials from published and evidence-based SST programmes for various disabilities as well as to include resources previously developed by the clinical team at the service for use with clients [2] [12] [13]. Ideas and resources were also adapted from an SST programme for children with hearing impairment developed at another Irish service.

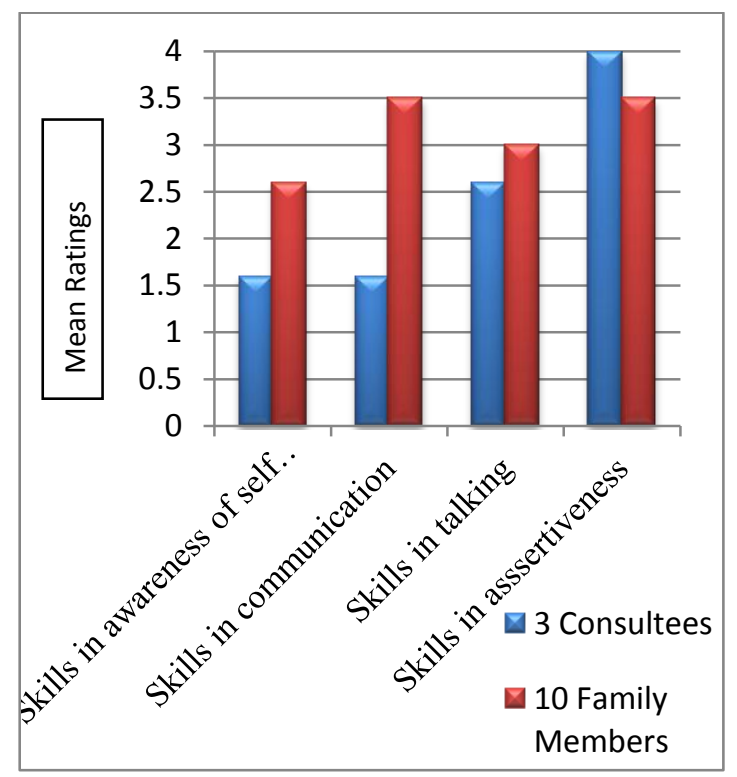

Figure 4. Mean ratings on social skills menu

In school consultation, teachers have been found to be less resistant and view interventions as more acceptable when interventions were considered to be effective, easily implemented and required little extra time or training [6]. The general format for sessions and a list of key principles to enhance generalisation of skills, are outlined in the introduction section of the manual [12] [13] [18]. The generation of a userfriendly package of SST for staff at the service was a key goal to increase treatment acceptability of the SST programme to increase the likelihood that SST services will be delivered to clients [20].The SST manual and participant workbook arising from this study is thereby used as a blueprint for future SST interventions at the service.

Multiple baseline measures were used to collect data from family members and clients themselves. Family members and the clients with ID completed the social domain of the Adaptive Behaviour Assessment System, Second Edition, Adult Form and the ABAS-II (Adult Self-Report Form, accessible version) respectively [8]. 
Table 1. Contents of SST manual

\begin{tabular}{|l|}
\hline Contents of SST sessions \\
\hline PART ONE: BASIC SKILLS \\
1. Introduction to SST, Identification of Feelings \\
\hline 2. Basic Greetings, Introducing Yourself \\
\hline 3. Starting Conversations \\
\hline 4. Topics of Conversation \\
\hline 5. Review Session \\
\hline 6. Compliments \\
\hline PART TWO: HIGHER-ORDER SKILLS \\
7. Conversation, Voice, Appropriate Social \\
Distance \\
\hline 8. Getting Ready for/joining Conversations \\
\hline 9. Understanding our Feelings, Signs of Upset \\
\hline 10. How to interrupt, Starting Conversations \\
\hline 11. How to Accept No, Ask Questions \\
\hline 12. Ending Conversations, Conflict Resolution \\
\hline 13. Talking about Problems \\
\hline 14. Touch, Friendships, Cheering up a friend \\
\hline 15. Sticking Up for Yourself \\
\hline 16. Asking Someone Out \\
\hline PART THREE: SST ASSESSMENT TOOLS \\
\hline$\bullet \quad$ Pre-intervention Interview \\
\hline$\bullet \quad$ Process-Evaluation (participants) \\
\hline$\bullet \quad$ Process-Evaluation (facilitators) \\
\hline$\bullet \quad$ Post-intervention Interview \\
\hline
\end{tabular}

2.4.4. Plan implementation. This stage involved implementing the intervention (technology training with consultees). Six session plans from the SST manual were co-directed by the researcher/consultant and main consultee, as part of technology training with staff. The remaining sessions are intended for use as future follow-on sessions with the current group and for use with future groups at the service. Clients were supported to choose their own aims (see table 2) for the group with Boardmaker visuals based on topics adapted from a published SST programme [12].

Table 2. Clients' aims for SST

\begin{tabular}{|l|c|}
\hline Aims & Frequency \\
\hline How to make friends & 5 \\
How to talk about feelings & 2 \\
How to speak more clearly & 2 \\
Keeping personal space & 2 \\
Staying calm in a crowd & 1 \\
How to ask for help & 1 \\
Talking about what I like & 1 \\
and what I don't like & 1 \\
\hline
\end{tabular}

A process-evaluation measure for facilitators (selfdesigned by the researcher using a five-point Likert scale) was used to monitor treatment integrity of each session and to prompt collaborative selfreflection between facilitators. Clients were supported by consultant/staff to complete formal process-evaluation measures to gain clients' views on programme design during this phase.

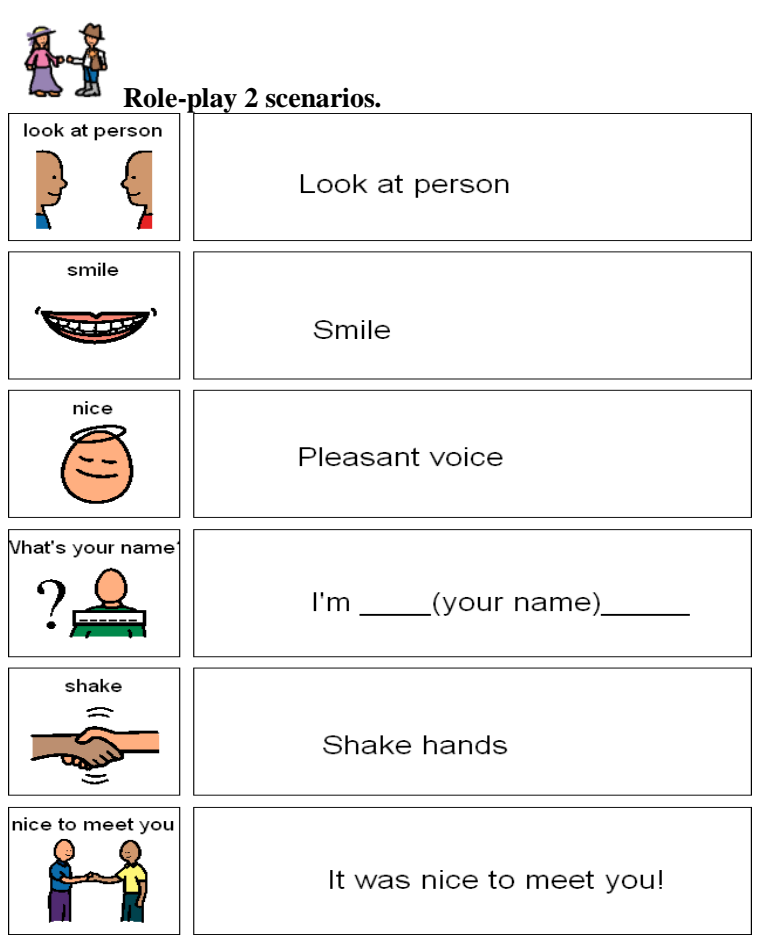

Figure 5. Sample item from client workbook

2.4.5. Plan evaluation. To assess programme efficacy, multiple baseline measures were taken with the social domain of the ABAS-II for triangulation of data with clients' self-reports [8]. Clients with ID completed the social domain of the ABAS-II, Adult Self-Report Form (accessible version) with supported decision-making procedures provided by lead researcher/staff to facilitate this process. Family members completed the social domain of the ABASII (Rated by Others form). Semi-structured questionnaires which focus on any changes seen in the specific target skills provide an opportunity for more socially valid results above and beyond what the ABAS-II can provide [4]. Family members were thus asked a few short open-ended questions that required them to detail specific changes, if any, that they have seen in their family member.

The Intervention Rating Profile - 15, a measure of treatment acceptability frequently used in school consultation, was adapted for use [15]. This involved minor changes to terminology (e.g. 'child' is changed to 'client') without any modifications to question-items [4]. All consultees completed the IRP-15 at post-intervention stage rating 15 evaluative statements regarding the extent to which the treatment is acceptable, appropriate and likely to be effective.

The Consultant Evaluation Form is widely used in school behavioural consultation research and is adapted for this adult education-setting [6]. This measure consists of 12 questions designed to 
evaluate consultee perceptions of a consultant and of the quality of the consultation. Items are rated on a 7point scale ranging from 'strongly disagree' to 'strongly agree'. To add social validity, the lead researcher conducted a semi-structured interview with the main consultee to capture her views of the SST programme beyond what rating scales can measure.

2.4.6. Ethical standards. This study was fully reviewed and approved by the School of Education Research Ethics Committee at University College Dublin. It adhered closely to policies and procedures for working with individuals with ID set out by the service psychological team, in particular to the means of gaining informed consent and measuring clients' views using supported decision-making processes.

\section{Results}

\subsection{Generalisation (ABAS-II: adult form)}

Results from family members' ratings on the social domain of the ABAS-II showed an improvement in social skills for eight out of ten clients. One client remained on the same score and another client's score was observed to decrease. The latter score was an outlier in the group, starting with the joint lowest score at pre-intervention and decreasing by 12 standard points. It is likely that this difference was influenced by interference effects. The Reliable Change Index (RCI) was used to ascertain whether or not changes observed were large enough to be attributable to the intervention rather than to measurement error [16]. As indicated in Table 3, there was a statistically reliable change from pre- to post- intervention $(\mathrm{p}<0.05)$ for six clients. Normative comparisons of ABAS-II data revealed that four out of six clients with statistically reliable change from pre- to post- intervention moved from one range to another, which indicates clinically significant changes in the social domain for four clients following consultation. Results suggest that $40 \%$ of clients with ID improved their social skills through the intervention.

\subsection{Questionnaires (families)}

Nine out of ten family members reported that their son/daughter enjoyed the intervention, learned a new skill and found the group helpful. Items relating to learning a specific new skill and usefulness of the group elicited responses, with six families providing short comments to describe the nature of changes observed. Daragh's family noted that he learned 'how to talk about his family' and Natasha's mother commented that 'she learned how to speak up for herself'.

Table 3: ABAS-II - Rated by Families

\begin{tabular}{|c|c|c|}
\hline \multirow{2}{*}{$\begin{array}{l}\text { Social } \\
\text { Domain } \\
\text { Family of: }\end{array}$} & \multicolumn{2}{|c|}{ Pre-Intervention } \\
\hline & Standard Score $^{\top}$ & Range \\
\hline Lucy & 72 & Borderline \\
\hline Julie & 81 & Below Average \\
\hline Jack & 66 & Extremely Low \\
\hline Ruth & 84 & Below Average \\
\hline Daragh & 66 & Extremely Low \\
\hline Natasha & 94 & Average \\
\hline Dominic & 66 & Extremely Low \\
\hline Monica & 75 & Borderline \\
\hline Barry & 72 & Borderline \\
\hline Neil & 70 & Extremely Low \\
\hline $\begin{array}{l}\text { Social } \\
\text { Domain }\end{array}$ & \multicolumn{2}{|c|}{ Post-Intervention } \\
\hline Family of: & Standard Score & Range \\
\hline Lucy & 75 & Borderline \\
\hline Julie & 84 & Below Average \\
\hline Jack & $54 \dagger$ & Extremely Low \\
\hline Ruth & $96 \dagger$ & Average $\ddagger$ \\
\hline Daragh & 68 & Extremely Low \\
\hline Natasha & $109+$ & Average \\
\hline Dominic & $75 \dagger$ & Borderline $\ddagger$ \\
\hline Monica & $92 \dagger$ & Average $\ddagger$ \\
\hline Barry & 72 & Borderline \\
\hline Neil & $78 \dagger$ & Borderline $\ddagger$ \\
\hline
\end{tabular}

Standard-score $\leq 70=$ Extremely Low; Standard-score 71-79 = Borderline; Standard-score 80-89 = Below Average; Standardscore 90-109 = Average;

Standard-score 110-119 = Above Average

$\uparrow$ Statistically reliable change.

† Clinically significant change

In relation to the nature of these changes, Ruth's father noted 'it's hard to tell if she learned a specific new skill but overall we have noticed an improvement' and Monica's father commented 'yes, she learned something but it's hard to say what'. This sentiment was echoed by Barry's parents also, who stated "yes [learned a new skill] but he cannot tell about it" and "we can just see that he [Barry] is being more sociable and willing to try new activities". Such comments may reflect the subtle nature of social skills and the difficulty with capturing specific changes in these skills [18].

\subsection{Client outcomes (ABAS-II: self-report)}

Results for clients' own ratings on the ABAS-II are not reported as part of the overall study but rather the ABAS-II was merely piloted as a measure with clients at this stage. The RCI was applied to track 
changes in clients' ratings of their perceived progress. However, limited conclusions could be drawn from the data and a lack of cross-form consistency showed family ratings of clients' social skills did not triangulate with clients' own ratings.

A number of external confounding factors may have given rise to non-interpretable results, which must be controlled for, where possible, in future studies. Firstly, individual assisted decision-making could not be provided to each client alone due to staff-constraints at the service. Instead, the ABAS-II was administered with clients in a small groupsetting, where clients could influence each other's answers. Clients may also be vulnerable to social desirability in their response-sets where they overstate their progress when rating themselves with familiar support-staff and by way of showing their enthusiasm for the intervention. Therefore, adapting and piloting the ABAS-II for clients with ID was merely a starting point in the development of a standardised tool to capture clients' own views in this area. As recognised in the literature, further research is required into the development of measures to capture the views of clients with ID as key stakeholders in relation to pertinent issues of well-being, quality of life and mental health [11].

\subsection{Process-evaluation (clients)}

Participants rated each session on a 4-point Likert scale for three simple statements about enjoyment, usefulness and difficulty (see figure 6). Data for session one was not included as this served to pilot the measure.

Overall, scores for Enjoyment $($ mean $=3.75)$ and Usefulness (mean $=2.95$ ) were high across sessions, suggesting participants enjoyed SST and acquired knowledge in each session. The mean score for Difficulty was lower throughout the programme $($ mean $=2.66)$, although a sharp increase after session two suggested participants felt challenged during subsequent sessions. This result prompted facilitators to run a review session for week five. The sixth session in the manual (compliments) was covered over two sessions, which appeared beneficial for clients with a reduction in the mean score for difficulty observed. Overall, progress evaluation measures served to inform appropriate pacing for clients.

An open-ended question followed each statement ("If yes, what did you enjoy? If yes, what was hard? If yes, what did you learn?") and responses were analysed qualitatively. In relation to enjoyment, props and activities emerged as a subtheme. After sessions, four clients mentioned the 'talking stick' and 'bean-bag' as enjoyable activities. Facilitation of interactions between group members contributed to the fun of the group. Daragh mentioned 'talking,' and 'we have to wait to talk' under 'Enjoyment' and
Barry noted he enjoyed 'talking about rugby and swimming'.

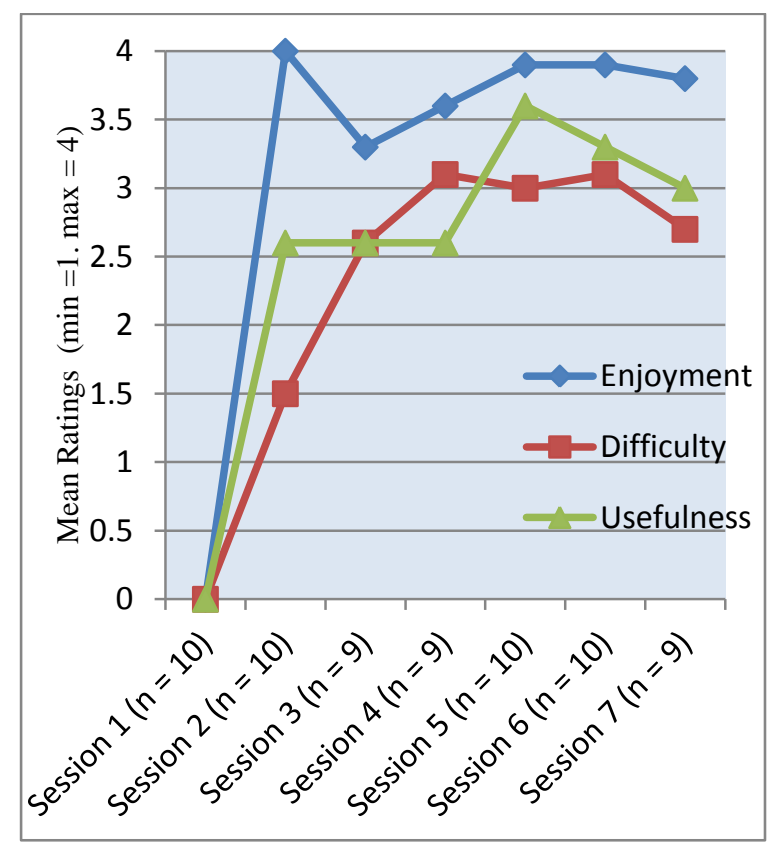

Figure 6. clients' process-evaluation data

Specific skills and activities also emerged as a subtheme of 'Usefulness'. 'Shaking hands' and 'sharing' were comments made, suggesting clients specifically remembered these skills from sessions. Similarly, many clients noted specific phrases as useful. After session seven, Jack remarked '[I will] say that's a nice garden to someone', which covered compliments.

Many comments made in later sessions reflected a subtheme of getting to know each other as an important aspect of the group for clients, suggesting that group cohesiveness formed as weeks passed. For example, Natasha commented that 'meeting Neil' and 'smiling' were useful aspects of session five. Monica noted that 'I learned about Natasha and her family' after session seven.

No clear pattern of subthemes emerged under the item for difficulty. Interestingly, Lucy mentioned the 'talking stick' as difficult after session two and mentioned it under 'Enjoyment' after session six. This suggests that consistent repetition and review with an activity that may be daunting at first, can yield positive results for individuals with ID.

\subsection{Treatment acceptability}

Responses to the IRP-15 indicated a high level of acceptability of the intervention (see table 4). Consultees rated the intervention with a total mean score of 84.3 with a range of $78-90$. 
Table 4: Intervention Rating Profile -15

\begin{tabular}{|l|c|}
\hline Item & $\begin{array}{c}\text { Post- } \\
\text { intervention } \\
\text { Mean item } \\
\text { ratings } \\
\text { (item range } \\
\mathbf{1 - 6 )}\end{array}$ \\
\hline 1. Acceptable Intervention & 5.5 \\
2. Generalisation of Intervention & 5.3 \\
3. Effective intervention & 5.5 \\
4. Refer intervention to others & 5.5 \\
5. Problem severity warranted & 5.3 \\
intervention & 5.5 \\
6. Suitable intervention & 5.8 \\
7. Willing to re-use intervention & 5.5 \\
8. No negative side effects & 5.8 \\
9. Appropriate for various groups & 5.5 \\
10. Consistency with other & 5.6 \\
11. Helpful intervention & 5.5 \\
12. Adaptable intervention & 5.8 \\
13. Like intervention procedures & 5.8 \\
14. Appropriate intervention & 6 \\
15. Intervention benefits clients & $\mathbf{8 4 . 3}$ \\
\hline Mean total score & \\
(max score = 90) & \\
N = 6 &
\end{tabular}

\subsection{Questionnaire (consultee)}

The questionnaire included a number of openended questions prompting reflection under the following themes: benefits for clients; benefits for the centre; and future use of the programme; which were analysed qualitatively for subthemes. The cofacilitator/consultee reported that the intervention would be 'rolled out' for future groups at the centre 'using the manual'. She highlighted that the programme will be run 'under the Communications module... at the centre'.

Under the master theme of benefits for clients, increased interaction among the client group emerged as a subtheme. The co-facilitator stated 'it [the programme] has got them talking' and expanded with 'they had some of the skills but they're more assertive about using their skills now'. She referred to the 'buzz of conversation among clients' she witnessed on the morning of the last session and described 'a restaurant-type atmosphere' in the room.

In relation to benefits for the centre, the cofacilitator emphasised autonomy for the centre as one of the major benefits by saying 'we [staff at the centre] don't have to depend on the clinical team, we can still look for advice but staff may not be released to help'.
When discussing future use of the programme, she highlighted the manual as useful because 'staff need the objectives to follow' and '...it's broken down into lesson plans which is very useful'. The cofacilitator emphasised her intention to follow the programme stating '[the staff] will do an assessment at the end of each section or at the end of every objective'. Qualitative data revealed benefits of the SST programme to clients and the centre that cannot be captured by rating scales such as the IRP.

\subsection{Consultant effectiveness}

Consultee responses to the Consultant Evaluation Form indicated high overall satisfaction with the consultation process (see Table 5). The consultant received a mean score of 52 with a range from 50 to 54 following implementation of the SST programme/technology training.

Table 5: Consultant Evaluation Ratings

\begin{tabular}{|l|c|}
\hline Item & $\begin{array}{c}\text { Mean Item rating } \\
\text { (item range 1-7) } \\
\text { (item range 1-7) }\end{array}$ \\
\hline 1 General helpfulness & 6.5 \\
2 Useful information & 6.5 \\
3 Good Listener & 6.25 \\
$\begin{array}{l}\text { 4 Useful resources } \quad \text { Fit well into } \\
\text { environment }\end{array}$ & 6.75 \\
6 Collaborative & 6.5 \\
7 Good Assistance & 6.75 \\
8 Would do it again & 6.5 \\
\hline $\begin{array}{l}\text { Mean score total } \\
\text { (max score =56) }\end{array}$ & 6.75 \\
$\mathbf{N}=\mathbf{6}$ & $\mathbf{5 2 . 5}$ \\
\hline
\end{tabular}

\section{Discussion}

The present study focused on designing a social skills training (SST) programme for adults with Intellectual Disability (ID) through a model of consultation. Examining the degree to which remedial and preventive goals of consultation were attained involved the collection of multiple measures in order to establish; 1) Client outcomes; 2) Consultees' attitudes toward the consultation process; 3) Consultees' attitudes toward the intervention and toward the possibilities for continuing the SST programme as part of on-going service delivery at the organisation. An SST manual of 230 pages, complete with session plans, materials and assessment resources, are a major outcome of the 
present study. A client workbook was also produced to accompany the programme. There was improvement to the service at a systemic-level in relation to SST with the empowerment of consultee personnel within the service to continue the intervention. The social domain of the ABAS-II was adapted into accessible format and piloted as a selfreport tool for adults with ID and remains at the service for use in future SST research.

In relation to generalisation of clients' social skills, results indicated moderate improvements in social skills to the home setting. Results from families on the ABAS-II showed that SST led to reliable and clinically significant improvements in clients' social skills for four out of ten clients after the first six sessions of the programme, which is a similar trend to results observed in research conducted in schools by Minihan et al [15]. Of clinical relevance, however, results suggest that $40 \%$ of the group may be ready to cover higher-order skills contained in part two of the programme, while half the group are likely to benefit from repeating introductory sessions again. Qualitative data indicated that gains were noticed by at least half of the families, contributing social validity to clients' ABAS-II scores. Qualitative data from the cofacilitator's completed questionnaire revealed a 'restaurant-type atmosphere' among clients during the final session. This suggests that subtle changes were observed above and beyond what could be captured in clients' ABAS-II scores. Given that only six sessions were delivered from the programme of sixteen sessions developed, further improvements in clients' social skills could be expected after implementation of the complete programme.

The programme followed guidelines for best practice in promoting generalisation of skills [20]. Many role-plays referred to situations in the home and community of relevance to clients' lives, as recommended in previous research [15]. Weekly homework activities were set from the client workbook to prompt use of skills between sessions, in line with recommendations from SST literature [7]. A number of barriers led to clients' inconsistent completion of homework activities such as the level of support clients required to meet a social skills buddy and to read task requirements. It was not always possible for the service to assign staff to supervise completion of homework activities. Practicing skills with a group of typical peers has led to improved social interactions in other studies [15] [19]. In the present study, the role-play assistant in attendance differed from week to week, constantly exposing clients to new social interactions reflective of work settings in the community. The inclusion of multiple peers without ID (i.e. more than just one role-play assistant) may have contributed towards greater generalisation effects for clients.
Process-evaluation measures served to gain clients' views on the programme after each session, taking a participatory approach to research as used in previous research for ID [14]. Clients' views on the process were used to inform pacing of the programme; e.g. a review session was incorporated into the programme for session five arising from clients' high ratings on difficulty after preceding sessions. Research suggests that people with ID tend to give responses that interviewers expect [9]. However, the mean score for 'Difficulty' was moderately low throughout the programme compared with scores for 'Enjoyment' and Usefulness', which indicates clients' differentiation between meanings of 'enjoy', 'useful' and 'difficult' in expressing their views and lends a degree of validity to clients' responses. Low 'difficulty' scores also suggest that a programme which is appropriate for clients' ability levels was achieved. Additionally, subthemes identified (e.g. Facilitation of interactions and getting to know each other) also suggest the intervention helped many clients achieve their common aim of learning 'how to make friends'. Given that 'lack of participation in activities' and 'impoverished social support' are identified as significant risk factors in developing depression for individuals with ID, the importance of an SST programme in helping clients make friends becomes apparent [10]. Furthermore, use of inclusive instructional practices (e.g. concrete materials, roleplays and cooperative-learning) endorsed by research were reflected in clients' responses under subthemes of props and activities and facilitation of interactions [19]. Taken together, these measures suggest that published SST curricula were adapted appropriately in the design of an SST programme that is accessible to individuals with ID, meeting a major goal of the present study.

\subsection{Strengths of Current Intervention}

The service has been provided with an SST Programme that has been specifically designed and adapted for persons with ID that can be used as a social skills intervention with other clients in the future. Resources were selected from published evidence-based programmes of SST [2] [12]. Research suggests the design and selection of interventions should be based on empirically supportable treatments [20]. The SST programme began with basic social skills identified by clients themselves (e.g. how to make friends) and progressed to higher-order skills (e.g. assertiveness) identified by staff and families. This is in line with findings in SST literature that skills should be covered in a hierarchical structure [12] [18]. Clinical interviews from published curricula were made accessible as pre- and post- assessment questionnaires, piloted with clients and included in 
the SST manual. According to the SST literature, drawing on clients' views is vital in the identification of their needs, which is in line with findings from quality of life literature [11] [12] [18]. The ABAS-II self- report questionnaire was adapted for use with clients with ID which will be a valuable resource as an evaluation tool in the future. Consultation including technology training was delivered, albeit an adapted and more extensive version of technology training than that used in school consultation models [6]. Research suggests that a clearly articulated model is important for increasing positive outcomes in instances of consultation [7].

In school consultation, the consultant usually carries out one or two training sessions with consultees with a view to consultees' subsequent delivery of the programme to clients [7]. To maximise treatment integrity in the present study, the consultant had direct involvement with consultees and clients in co-delivering six pilot sessions from the SST programme. This provides opportunities for the researcher to thoroughly cover issues of resources, materials and training with consultees and to empower staff for on-going implementation of the programme thereafter. To adhere to treatment integrity of the programme, facilitators rated their skills after each session based on ten core principles recommended from SST literature [12] [13]. This checklist was included in the SST manual. Previous research recommended use of checklists in documenting facilitators' mastery of skills during technology training [15].

\subsection{Limitations}

This study has a number of limitations. Factors that may influence informant-ratings were not controlled for in the present study, such as affective changes or parents' literacy skills, which may have impacted on informants' capacity or willingness to complete ABAS-II questionnaires. Random selection of the sample was not possible in the present study because the service prioritised clients based on SST needs. The waiting group could have been used as a control group but this was not possible due to staff limitations. Confidentiality was explained to clients and examination-type conditions were applied for the completion of process-evaluation measures. Despite this, clients may have influenced each other's answers in the small-group setting. Researchers must be aware that clients with ID are subject to social desirability in their response-sets.

\section{Conclusion}

Clients' views were taken into account in the development of this SST programme, reflecting a participatory approach to research and one that is responsive to clients' needs. Findings suggest that preventive goals of consultation were attained and that the SST manual and participant workbook will be used as a blueprint for future interventions at the service. Results showed moderate generalisation of clients' social skills to the home setting. This suggests clients may require repetition and review of pilot sessions and/or completion of the full SST programme of sixteen session plans before the remedial goals of consultation can be fully realised.

The core outcomes of the study include an improvement to the service at a systemic-level, in relation to SST, the empowerment of consultee personnel within the service and improvement in social skills of clients who participated in the programme, with the further potential to enhance their social skills and those of other clients who may access the programme in the future. Results suggest that this instance of collaborative-work through consultation shifted the researcher's role beyond one of designing and implementing an SST programme for merely one group of clients. The present study contributes to the field of ID and consultation research as it supports the application of an Educational Psychologist's skills to a service that supports adults with ID.

\section{Acknowledgement}

We would like to thank all the staff at the training centres at the service and members of the clincal team who consulted with us. We would like to especially thank fourteen clients for their enthusiastic participation in the social skills sessions during the development of this training programme.

\section{References}

[1] American Psychiatric Association: Diagnostic and Statistical Manual of Mental Disorders, Fifth Edition. Arlington, VA, American Psychiatric Association, 2013. Web. [access date: 1 June 2013]. dsm.psychiatryonline.org

[2] Baker, J. E., Social Skills Training: For Children and Adolescents with Asperger Syndrome and SocialCommunication Problem, Autism Asperger Publishing Co., Kansas, 2003.

[3] Carr, A., O' Reilly, G., Noonan-Walsh, P., \& McEvoy, J., The Handbook of Intellectual Disability and Clinical Psychology Practice. Routledge, New York: 2007.

[4] Cohen, L., Manion, L., \& Morrison, K., Research Methods in Education (7th edn.)., Routledge, New York, 2011.

[5] Department of Health. (2005). Mental Capacity Act. London: Her Majesty's Stationery Office. 
[6] Erchul, W. \& Sheridan, S., Handbook of Research in School Consultation, Taylor \& Francis Group/Routledge, New York, 2008.

[7] Gresham, F. (2002). "Best Practices in Social Skills Training". In A. Thomas, \& J. Grimes, Best Practices in School Psychology $V$, The National Association of School Psychologists, Bethesda, M.D, pp. 1029-1040.

[8] Harrison, \& Oakland, The Adaptive Behaviour Assessment System-Second Edition (ABAS-II), Pearson Assessments, San Antonio, 2003.

[9] Hayashi, M., Arakida, M., \& Kazumoto, O., "The effectiveness of a sex education program facilitating social skills for people with intellectual disability in Japan", Journal of Intelllectual \& Developmental Disability, 36 (1), 2011, pp. 11-19.

[10] Kearney, D., \& Healy, O., Investigating the relationship between challenging behavior, co-morbid psychopathology and social skills in adults with moderate to severe intellectual disabilities in Ireland. Research in Developmental Disabilities, 32, 2011, pp.1556-1563.

[11] Keith, K., Quality of Life. In A. Carr, G. O'Reilly, P. Noonan-Walsh, \& J. McEvoy, The Handbook of Intellectual Disability and Clinical Psychology Practice, Routledge, New York, 2007, pp. p.143-168.

[12] Kelly, A., Talkabout: Developng Social Communication Skills. Speechmark, UK, 1996, 2000.

[13] Kingsep, P., \& Nathan, P., Social Skills Training for Severe Mental Disorders: A Therapist's Manual. Centre for Clinical Interventions, Perth, 2003.

[14] McGillivray, J. A., \& McCabe, M. P., "Detecting and treating depression in people with mild intellectual disability: the views of key stakeholders", British Journal of Learning Disabilities, 38, 2009, pp.68-76.

[15] Minihan, A., Kinsella, W., \& Honan, R., "Social skills training for adolescents with Asperger's syndrome using a consultation model", Journal of Research in Special Educational Needs, 11, 2011, pp.55-69.

[16] Moleiro, C., \& Beutler, L., "Clinically significant change in psychotherapy for depressive disorders". Journal of Affective Disorders, 2009, pp. 220-224.

[17] Sigafoos, J., O'Reilly, M., \& Green, V., "Communication difficulties and the promotion of communication skills". In A. Carr, G. O'Reilly, P. Noonan Walsh, \& J. McEvoy, The Handboook of Intellectual Disability and Clinical Psychology Practice, Routledge, New York, 2007, pp. 607-642.

[18] Spence, S., "Social Skills Training with Children and Young People: Theory, Evidence and Practice". Child and Adolescent Mental Health, 8, 2003, pp. 84-96.

[19] Wehmeyer, M., \& Lee, S.-H., "Educating Children with Intellectual Disability and Autism-Spectrum Disorders". In A. Carr, G. O'Reilly, P. Noonan-Walsh, \& J. McEvoy, The Handbook of Intellectual Disability and
Clinical Psychology Practice, Routledge, New York, 2007, pp. 559-605.

[20] Zins, J., \& Erchul, W., Best practices in school consultation. US National Association of School Psychologists, Washington, DC, 2002. 